\title{
Magnetic condensation and confinement in lattice gauge theory
}

\author{
P. Cea ${ }^{\mathrm{a}, \mathrm{b}}$, and L. Cosmai ${ }^{\mathrm{b}}$

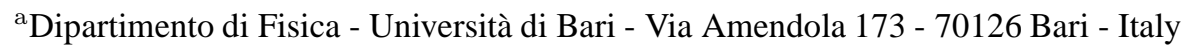 \\ ${ }^{\mathrm{b}}$ INFN - Sezione di Bari - Via Amendola 173 - 70126 Bari - Italy
}

We investigate Abelian monopoles and Abelian vortices condensation in finite temperature SU(2) and SU(3) pure lattice gauge theories.

\section{INTRODUCTION}

To study the vacuum structure of lattice gauge theories we introduced [1.2] a gauge invariant effective action, defined by using the lattice Schrödinger functional

$$
\mathcal{Z}\left[U_{\mu}^{\text {ext }}\right]=\int \mathcal{D} U e^{-S_{W}},
$$

where $S_{W}$ is the standard Wilson action and the functional integration is extended over links on a lattice with the hypertorus geometry and satisfying the constraints

$$
\left.U_{\mu}(x)\right|_{x_{4}=0}=U_{\mu}^{\mathrm{ext}}(\vec{x}) .
$$

The same constraint also applies to the links exiting from sites belonging to the spatial boundaries (this last condition corresponds to require that the fluctuations over the background field vanish at infinity). The lattice effective action for the external static background field $U^{\text {ext }}(\vec{x})$ is

$$
\Gamma\left[U^{\mathrm{ext}}\right]=-\frac{1}{L_{4}} \ln \left\{\frac{\mathcal{Z}\left[U^{\mathrm{ext}}\right]}{\mathcal{Z}(0)}\right\},
$$

$L_{4}$ is the extension in Euclidean time, $\mathcal{Z}(0)$ is the lattice Schrödinger functional, without the external background field $\left(U_{\mu}^{\text {ext }}=1\right)$. It can be shown that: $\Gamma\left[U^{\text {ext }}\right]$ is invariant for lattice gauge transformations of the external links $U_{\mu}^{\text {ext }}$, and $\lim _{T \rightarrow \infty} \Gamma\left[U^{\text {ext }}\right]=$ $E_{0}\left[U^{\text {ext }}\right]-E_{0}[0], E_{0}\left[U^{\text {ext }}\right]$ vacuum energy in presence of the external background field.

At finite temperature, we consider the thermal partition function in presence of a given static back- ground field:

$$
\mathcal{Z}_{T}\left[U^{\mathrm{ext}}\right]=\int_{U_{k}\left(\beta_{T}, \vec{x}\right)=U_{k}(0, \vec{x})=U_{k}^{\mathrm{ext}}(\vec{x})} \mathcal{D} U e^{-S_{W}} .
$$

Now the temporal links obey p.b.c.'s while spatial links belonging to the time slice $x_{4}=0$ or exiting from sites belonging to the spatial boundaries are constrained to $U_{k}\left(\vec{x}, x_{4}\right)=U_{k}^{\text {ext }}(\vec{x})$. After sending the physical temperature to zero the thermal functional Eq. (4) reduces to the zerotemperature Schrödinger functional with the constraints $\left.U_{k}(x)\right|_{x_{4}=0}=U_{k}^{\text {ext }}(\vec{x})$.

To investigate Abelian monopoles and Abelian vortices condensation we make use of a disorder parameter [ [3-5] defined in terms of the thermal partition functional Eq. ( (4) in presence of, respectively, an external Abelian monopole or Abelian vortex background field. Since our thermal partition functional is constructed by means of the Schrödinger functional which is invariant against gauge transformations of the background field we do not need to do any gauge fixing to perform the Abelian projection. Indeed, after choosing the type of Abelian monopoles, our results do not depend on the particular direction selected in the color space, which, actually, can be varied by a gauge transformation.

\section{ABELIAN MONOPOLE CONDENSATION}

\section{1. $\mathrm{SU}(2)$}

We put on the background the lattice version of the Abelian monopole field

$$
g \vec{b}^{a}(\vec{x})=\delta^{a, 3} \frac{n_{\text {mon }}}{2} \frac{\vec{x} \times \vec{n}}{|\vec{x}|(|\vec{x}|-\vec{x} \cdot \vec{n})},
$$


$\vec{n}$ is the direction of the Dirac string and, according to the Dirac quantization condition, $n_{\text {mon }}$ is an integer. The lattice links corresponding to the Abelian monopole field Eq. (5) can be readily obtained as:

$$
U_{k}^{\mathrm{ext}}(\vec{x})=\mathrm{P} \exp \left\{i g \int_{0}^{1} d t \frac{\sigma_{a}}{2} b_{k}^{a}\left(\vec{x}+t \hat{x}_{k}\right)\right\},
$$

where the $\sigma_{a}$ 's are the Pauli matrices. By choosing $\vec{n}=\hat{x}_{3}$ we get:

$$
\begin{aligned}
& U_{1,2}^{\mathrm{ext}}(\vec{x})=\cos \left[\theta_{1,2}(\vec{x})\right]+i \sigma_{3} \sin \left[\theta_{1,2}(\vec{x})\right], \\
& U_{3}^{\text {ext }}(\vec{x})=\mathbf{1},
\end{aligned}
$$

with

$$
\begin{aligned}
& \theta_{1}(\vec{x})=-\frac{n_{\text {mon }}}{4} \frac{\left(x_{2}-X_{2}\right)}{\left|\vec{x}_{\text {mon }}\right|} \frac{1}{\left|\vec{x}_{\text {mon }}\right|-\left(x_{3}-X_{3}\right)}, \\
& \theta_{2}(\vec{x})=+\frac{n_{\text {mon }}}{4} \frac{\left(x_{1}-X_{1}\right)}{\left|\vec{x}_{\text {mon }}\right|} \frac{1}{\left|\vec{x}_{\text {mon }}\right|-\left(x_{3}-X_{3}\right)} .
\end{aligned}
$$

In Equation (8) $\left(X_{1}, X_{2}, X_{3}\right)$ are the monopole coordinates and $\vec{x}_{\text {mon }}=(\vec{x}-\vec{X})$.

We evaluate numerically the disorder parameter for confinement

$$
\mu=e^{-F_{\text {mon }} / T_{\text {phys }}}=\frac{\mathcal{Z}_{T}\left[n_{\text {mon }}\right]}{\mathcal{Z}_{T}[0]} .
$$

In Eq.(9) $\mathcal{Z}_{T}[0]$ is the thermal partition function without monopole field (i.e. with $n_{\text {mon }}=0$ ). From Eq. (9) it is clear that $F_{\text {mon }}$ is the free energy to create an Abelian monopole. Therefore in presence of monopole condensation $F_{\text {mon }}=0$ and $\mu=1$. Actually we consider the derivative of the monopole free energy $F_{\text {mon }}^{\prime}=\partial F_{\text {mon }} / \partial \beta$, It can be shown that $(V$ is the spatial volume)

$$
F_{\text {mon }}^{\prime}=V\left[<P l>_{n_{\text {mon }}=0}-<P l>_{n_{\text {mon }} \neq 0}\right] .
$$

$<P l>$ is the average plaquette. Figure 1 displays $F_{\text {mon }}^{\prime}$ vs. $\beta$ together with the absolute value $|P|$ of the Polyakov loop for a $24^{3} \times 4$ lattice. The peak in $F_{\text {mon }}^{\prime}$ corresponds to the rise of $\langle|P|\rangle$ at the deconfinement transition. The disorder parameter $\mu$ (Eq. (9)) can be obtained by a numerical integration in $\beta$ of the monopole free energy derivative. It follows that $\mu=1$ in the confined phase (i.e. the monopoles condense in the vacuum). Moreover, by increasing the

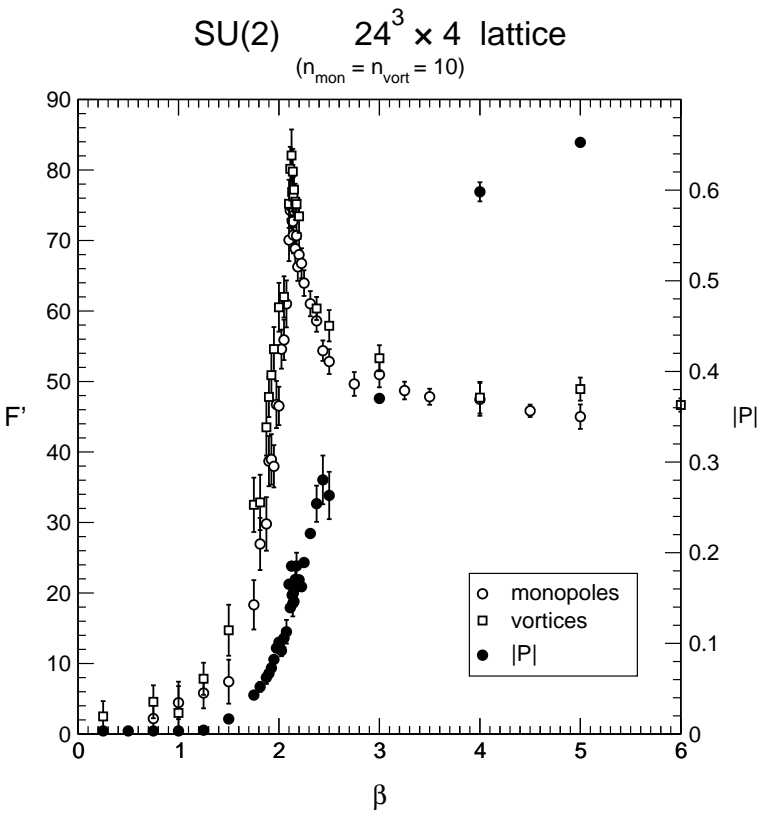

Figure 1. $F^{\prime}$ for monopoles (open circles) and vortices (squares) together with the absolute value of the Polyakov loop (full circles).

spatial volume of the lattice, the disorder parameter $\mu$ decreases faster toward zero in the deconfined phase, suggesting that $\mu \rightarrow 0$ in the thermodynamic limit when $\beta$ reaches the critical value.

\section{2. $\mathrm{SU}(3)$}

In the case of SU(3) gauge theory, the maximal Abelian group is $\mathrm{U}(1) \times \mathrm{U}(1)$. We have two different types of Abelian monopole. The first one is defined by Eq. (5) and we call it $T_{3}$ Abelian monopole. The lattice links are given by

$$
U_{1,2}^{\mathrm{ext}}(\vec{x})=\left[\begin{array}{ccc}
e^{i \theta_{1,2}(\vec{x})} & 0 & 0 \\
0 & e^{-i \theta_{1,2}(\vec{x})} & 0 \\
0 & 0 & 1
\end{array}\right],
$$

with $\theta_{1,2}(\vec{x})$ as in Eq. (8). The second type of independent Abelian monopole can be obtained by con- 
sidering the diagonal generator $\lambda_{8}$ :

$$
U_{1,2}^{\mathrm{ext}}(\vec{x})=\left[\begin{array}{ccc}
e^{i \theta_{1,2}(\vec{x})} & 0 & 0 \\
0 & e^{i \theta_{1,2}(\vec{x})} & 0 \\
0 & 0 & e^{-2 i \theta_{1,2}(\vec{x})}
\end{array}\right],
$$

with

$\theta_{1}(\vec{x})=\frac{1}{\sqrt{3}}\left[-\frac{n_{\text {mon }}}{4} \frac{\left(x_{2}-X_{2}\right)}{\left|\vec{x}_{\text {mon }}\right|} \frac{1}{\left|\vec{x}_{\text {mon }}\right|-\left(x_{3}-X_{3}\right)}\right]$,

$\theta_{2}(\vec{x})=\frac{1}{\sqrt{3}}\left[+\frac{n_{\text {mon }}}{4} \frac{\left(x_{1}-X_{1}\right)}{\left|\vec{x}_{\text {mon }}\right|} \frac{1}{\left|\vec{x}_{\text {mon }}\right|-\left(x_{3}-X_{3}\right)}\right]$.

We call it the $T_{8}$ Abelian monopole. The corresponding continuum gauge field is now

$$
g \vec{b}^{a}(\vec{x})=\delta^{a, 8} \frac{n_{\text {mon }}}{2} \frac{\vec{x} \times \vec{n}}{|\vec{x}|(|\vec{x}|-\vec{x} \cdot \vec{n})} .
$$

Other Abelian monopoles can be generated by considering the linear combination of the $T_{3}$ and $T_{8}$ generators. For instance

$$
T_{3 a}=-\frac{1}{2} \frac{\lambda_{3}}{2}+\frac{\sqrt{3}}{2} \frac{\lambda_{8}}{2}=\left[\begin{array}{ccc}
0 & 0 & 0 \\
0 & \frac{1}{2} & 0 \\
0 & 0 & -\frac{1}{2}
\end{array}\right] .
$$

Figure 2 shows that the $T_{8}$ Abelian monopole displays a signal about a factor two higher in the peak region. This result suggests that in the pattern of dynamical symmetry breaking due to the Abelian monopole condensation the color direction 8 is slightly preferred. Like for $\mathrm{SU}(2), F_{\text {mon }}^{\prime}$ displays a sharp peak in correspondence of the rise of the Polyakov loop, and, by increasing the spatial volume the peak increases. We find that the disorder parameter $\mu$ is different from zero in the confined phase and decreases towards zero in the thermodynamic limit at the critical coupling. Finally a qualitative analysis of $\mu$ suggests that the finite volume behavior is consistent with a first order phase transition [5].

\section{VORTEX CONDENSATION}

\section{1. $\mathrm{SU}(2)$}

We consider the continuum gauge field for a vortex directed along the $\hat{z}$-direction with $n$ units of elemen-
SU(3) Abelian monopoles

$32^{3} \times 4$ lattice $\left(\mathrm{n}_{\text {mon }}=10\right)$

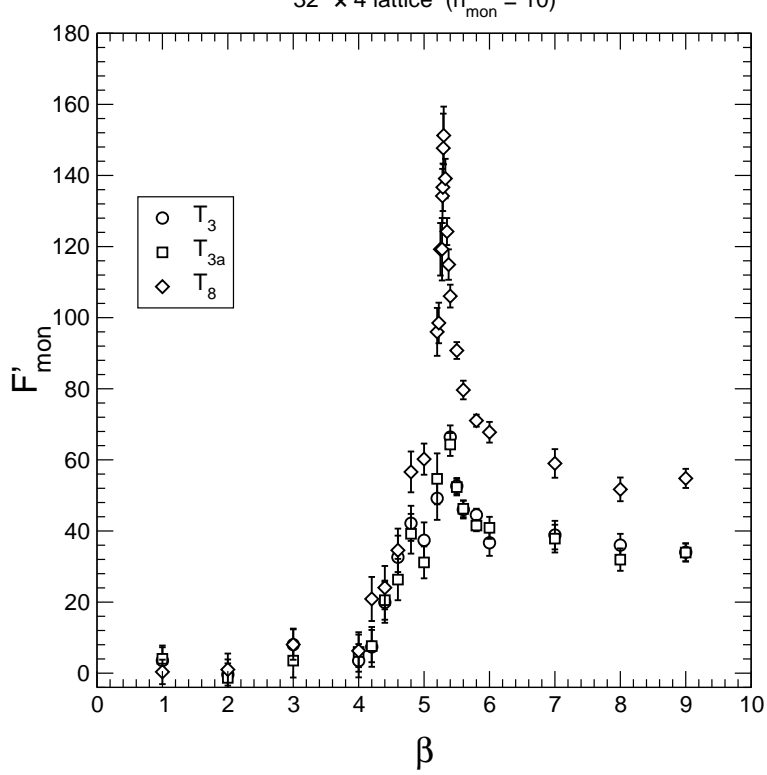

Figure 2. $\quad F_{\mathrm{mon}}^{\prime}$ for $T_{3}$ monopoles (circles), $T_{3 a}$ monopoles (squares) and $T_{8}$ monopoles (diamonds).

tary flux $\phi=\frac{2 \pi}{g}$ is given by

$$
\begin{aligned}
A_{x}^{\mathrm{ext}} & =-\frac{n}{g} \frac{x_{2}}{\left(x_{1}\right)^{2}+\left(x_{2}\right)^{2}}, \\
A_{y}^{\mathrm{ext}} & =\frac{n}{g} \frac{x_{1}}{\left(x_{1}\right)^{2}+\left(x_{2}\right)^{2}}, \\
A_{z}^{\mathrm{ext}} & =A_{0}=0 .
\end{aligned}
$$

On the lattice

$$
\begin{aligned}
& U_{1,2}^{\text {ext }}(\vec{x})=\cos \left[\theta_{1,2}(\vec{x})\right]+i \sigma_{3} \sin \left[\theta_{1,2}(\vec{x})\right], \\
& U_{3}^{\text {ext }}(\vec{x})=\mathbf{1}, \\
& \theta_{1,2}(\vec{x})=\mp \frac{n_{\text {vort }}}{2} \frac{x_{2,1}}{\left(x_{1}\right)^{2}+\left(x_{2}\right)^{2}}
\end{aligned}
$$

We evaluate $F_{\text {vort }}^{\prime}=\partial F_{\text {vort }} \partial \beta$, where $F_{\text {vort }}$ is the free energy to create an Abelian vortex $F_{\text {vort }}$. This amounts to compute

$$
F_{\text {vort }}^{\prime}=V\left[<P l>_{n_{\text {vort }}=0}-<P l>_{n_{\text {vort }} \neq 0}\right] .
$$

Figure 1 shows that the free energy for both monopoles and vortices displays a peak in correspon- 
dence of the finite temperature deconfinement transition suggesting that vortices play a role in the dynamics of the deconfinement transition (in qualitative agreement with Ref. [6]).

\section{2. $\mathrm{SU}(3)$}

We have considered two different kinds of vortices. Namely the $T_{8}$ vortex:

$$
\begin{aligned}
U_{1,2}^{\mathrm{ext}}(\vec{x}) & =\left[\begin{array}{ccc}
e^{i \theta_{1,2}(\vec{x})} & 0 & 0 \\
0 & e^{i \theta_{1,2}(\vec{x})} & 0 \\
0 & 0 & e^{-2 i \theta_{1,2}(\vec{x})}
\end{array}\right], \\
U_{3}^{\operatorname{ext}}(\vec{x}) & =\mathbf{1} \\
\theta_{1,2} & =\mp \frac{1}{\sqrt{3}} \frac{n_{\text {vort }}}{2} \frac{x_{2,1}}{\left(x_{1}\right)^{2}+\left(x_{2}\right)^{2}}
\end{aligned}
$$

and the $T_{3}$ vortex:

$$
\begin{aligned}
U_{1,2}^{\text {ext }}(\vec{x}) & =\left[\begin{array}{ccc}
e^{i \theta_{1,2}(\vec{x})} & 0 & 0 \\
0 & e^{-i \theta_{1,2}(\vec{x})} & 0 \\
0 & 0 & 1
\end{array}\right], \\
U_{3}^{\text {ext }}(\vec{x}) & =\mathbf{1} \\
\theta_{1,2} & =\mp \frac{n_{\text {vort }}}{2} \frac{x_{2,1}}{\left(x_{1}\right)^{2}+\left(x_{2}\right)^{2}}
\end{aligned}
$$

Also in this case we clearly see (Fig. 3) a peak in $F_{\text {vort }}^{\prime}$ corresponding to the rise of the absolute value of the Polyakov loop at the deconfinement transition. As in the case of Abelian monopoles we find that $F_{\text {vort }}^{\prime}$ for the $T_{8}$ vortex gives a stronger signal than the corresponding quantity for the $T_{3}$ vortex.

\section{CONCLUSIONS}

We investigated the condensation of Abelian monopoles and Abelian vortices in the finite temperature $\mathrm{SU}(2)$ and $\mathrm{SU}(3)$ lattice gauge theories using a disorder parameter defined in terms of a lattice thermal partition functional. Our numerical results suggest that monopoles and vortices condense in the vacuum of non Abelian 1.g.t.'s., in agreement with analogous results obtained using different approaches [ד].

Let us conclude by stressing that our method, while keeping the gauge invariance, can be readily extended to study the effect of the dynamical fermions.

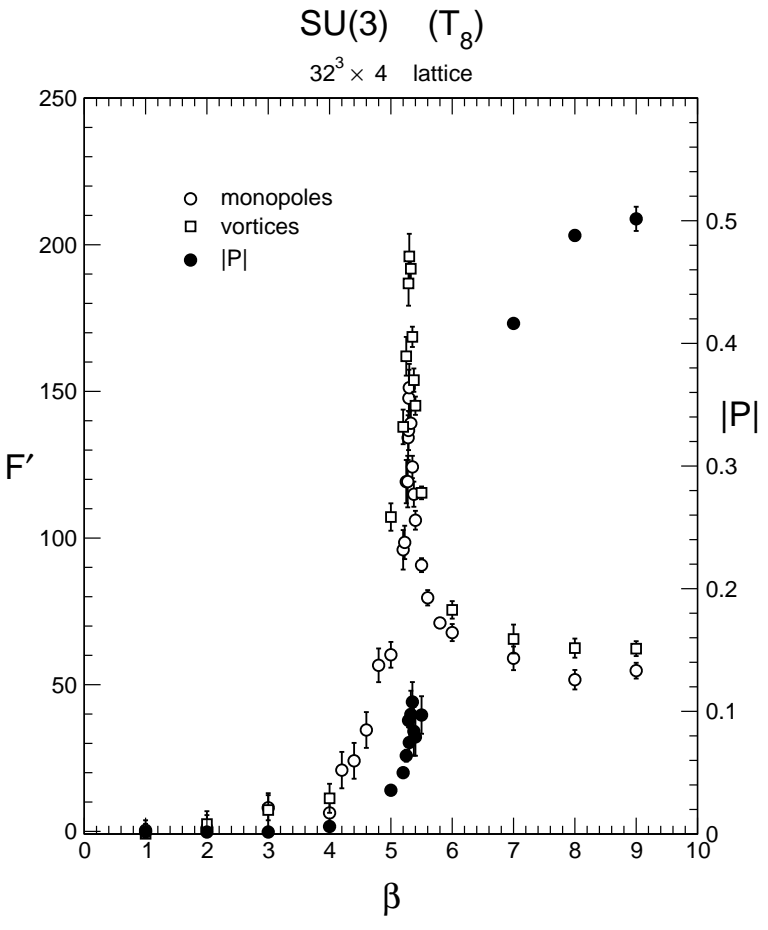

Figure 3. $F^{\prime}$ for monopoles (open circles) and vortices (squares) together with the absolute value of the Polyakov loop (full circles).

\section{REFERENCES}

1. P. Cea and L. Cosmai, in Nucl. Phys. Proc. Suppl. 53 (1997) 574.

2. P. Cea and L. Cosmai, Phys. Rev. D60 (1999) 094506.

3. L. Del Debbio, A. Di Giacomo and G. Paffuti, Phys. Lett. B349 (1995) 513.

4. A. Di Giacomo, B. Lucini, L. Montesi and G. Paffuti, Phys. Rev. D61 (2000) 034503; ibid. D61 (2000) 034504.

5. P. Cea and L. Cosmai, Phys. Rev. D62 (2000) 094510.

6. L. Del Debbio, A. Di Giacomo and B. Lucini, hep-lat/0006028.

7. see, for instance, the session "Topology and Vacuum" of these Proceedings. 